\title{
Los niveles de actuación en las relaciones de trabajo y la formación de contratos psicológicos*
}

\author{
The levels of action in work relationships and \\ the formation of psychological contracts
}

\author{
Erico Rentería Pérez \\ ORCID: 0000-0002-0538-925X \\ Universidad del Valle, Colombia \\ Universidade Federal da Bahía, Brasil \\ Juan Javier Vesga Rodríguez** \\ ORCID: 0000-0003-2382-5104 \\ Universidad Católica de Colombia \\ Recibido: 17 de febrero de 2018 \\ Revisado: 6 de abril de 2018 \\ Aceptado: 4 de junio de 2018
}

\section{Resumen}

El contrato psicológico es un concepto clave en la comprensión de las relaciones de trabajo en la actualidad. Sin embargo, dada la pluralidad y composición de éstas por las dinámicas empresariales, la concepción sobre la formación de contratos psicológicos se hace a la vez compleja. En este sentido, se propone en este artículo teórico, el análisis de la estructuración de las relaciones de trabajo a través de diversos niveles de actuación, como encuadre para la comprensión de la formación de contratos psicológicos. Esta perspectiva permite obtener una visión más amplia y completa de la manera como funcionan las relaciones de trabajo, con el propósito de adoptar una mirada multidimensional del contrato psicológico, para poder entender que éste no se reduce a una relación diádica, sino que en su formación intervienen otros aspectos provenientes de los diversos niveles en que se configuran las relaciones de trabajo en las organizaciones.

Palabras clave: contrato psicológico, relaciones de trabajo, psicología organizacional y del trabajo, comportamiento organizacional.

Artículo teórico. Citar como: Rentería, P. E. \& Vesga, R. J. J. (2019). Los niveles de actuación en las relaciones de trabajo y la formación de contratos psicológicos. Diversitas: Perspectivas en Psicología, 15(1), 129-142. DOI: https://doi.org/10.15332/s1794-9998.2019.0001.10

Correspondencia: Juan Javier Vesga, Universidad Católica de Colombia. Dirección postal: Avda. Caracas \# 46-40. Correo electrónico: jjvesga@ ucatolica.edu.co 


\section{Abstract}

The psychological contract is a key concept for understanding work relationships today. However, given the plurality and composition of these by business dynamics, the conception of the formation of psychological contracts becomes complex too. In this sense, current theoretical article proposes the analysis of the structuring of labor relations through different levels of action, as a framework for the understanding of the formation of psychological contracts. This perspective allows us to obtain a wider and more complete vision of how the working relationships work, all of this in order to adopt a multidimensional view of the psychological contract, in order to understand that it doesn't reduce to a dyadic relationship, but in its formation other aspects intervene from the various levels in which work relationships are configured in organizations.

Keywords: psychological contract, work relationships, work and organizational psychology, organizational behavior.

\section{Introducción}

El Contrato Psicológico (CP) es un concepto de gran relevancia en la literatura de la Psicología Organizacional y del Trabajo (POT) en la actualidad. Diversos autores coinciden en destacar la importancia que tiene este concepto para el análisis y comprensión de la forma como se construyen actualmente los vínculos laborales entre trabajadores y organizaciones (Alcover, Rico, Turnley, \& Bolino, 2016; Malvezzi, 2011; Topa, 2005, entre otros).

El CP se define como el conjunto de compromisos implícitos en la relación de trabajo, más allá de los acuerdos formales explícitos establecidos entre las partes en el marco de un acuerdo de relación laboral. En general, entre los investigadores y teóricos se acepta la importancia que tiene este concepto como recurso para la comprensión de las relaciones de trabajo. Para Topa (2005) el CP "propone un acercamiento prometedor a la relación laboral desde una perspectiva psicosocial" (p. 102). Por su parte, Coyle-Shapiro y Parzefall (2010) indican que su estudio ha atraído la atención de investigadores como una estructura o sistema para el entendimiento de las relaciones de trabajo, ya que las relaciones de trabajo no están reducidas sólo a las condiciones pactadas en el contrato jurídico, sino que incluyen toda una variedad de percepciones, expectativas, creencias, conocimientos y experiencias acerca de los compromisos implícitos y explícitos no oficializados en la relación de trabajo (Alcover, 2002; Topa, 2005; Vesga, 2009), y "el concepto de CP intenta recoger todas estas realidades que escapan a la descripción reducida de la relación de empleo que consta en el contrato jurídico" (Topa, 2005, p. 102).

El CP se configura en el marco de las relaciones de trabajo. Un abordaje para su estudio ha sido la perspectiva jurídica, y es en este contexto que las mismas han sido más claramente definidas (Spooner \& Haidar, 2006). Sin embargo, aunque la existencia de una relación de trabajo está referida principalmente por los acuerdos formales establecidos entre la organización contratante y el trabajador, el asunto no se reduce exclusivamente al orden de lo jurídico en el que las partes involucradas -trabajador y organización- establecen una serie de acuerdos en el marco de las leyes que rigen los aspectos laborales en cada país.

Una relación comprende vínculos que se establecen entre dos o más partes de un sistema. En asuntos humanos, implica el establecimiento de interacciones y conexiones entre personas o grupos de personas a través de los cuales ellas se conocen, hacen intercambios, se comunican y generan acciones o espacios comunes. En este sentido, el concepto de relaciones de trabajo refiere los contactos e 
interconexiones que existen entre personas y entre éstas y las organizaciones de las que hacen parte, con ocasión de la realización de actividades de trabajo, entendiendo éste último como "una actividad económico-productiva” (Rentería, 2008, p. 69). Así, las relaciones de trabajo revisten una complejidad de miradas y posibilidades de análisis, ya que están definidas tanto por aspectos de carácter jurídico, económico y político, como psicosocial.

Un abordaje psicosocial de las relaciones de trabajo puede verse desde la perspectiva de Petit (1984), para quien "el objeto de la psicosociología es el estudio de los fenómenos de la interacción social entre individuos, entre individuos y grupos, y entre grupos [cursivas en el original]" (p. 13) en el contexto específico y cotidiano de una organización. Esta es vista como un sistema abierto, compuesto de diferentes elementos articulados y en continua interacción con el ambiente y el individuo, y los grupos como actores cuyo accionar no está determinado sólo por motivaciones económicas, impulsos afectivos y normas grupales, sino que son asumidos como actores en este sistema, agentes complejos y reflexivos (Rentería, 2008; Spink, 1996).

Una perspectiva psicosocial de las relaciones de trabajo comprende tres elementos fundamentales: el trabajador como actor social, la organización como sistema abierto y la interacción de estos dos a través de procesos de comunicación y relaciones de poder (Petit, 1984), en el que "lo psicosocial" no se refiere simplemente a una dualidad conformada por dos elementos aislados, individuo y organización, sino que alude a una dimensión integrada e integradora, es decir, un punto de convergencia entre el sujeto -incluyendo su historia y singularidad-, y los contextos laborales -situados a su vez en otros más amplios- en los cuales participa como sujeto de acción, o como actor (Petit, 1984).

Las relaciones de trabajo se configuran a través de diferentes niveles de actuación y complejidades en los que se estructuran, mantienen o modifican las dinámicas de trabajo, tal como ha sido propuesto por autores como Barnard (1968), Petit (1984), Schein (1994), Spink (1996), Rentería \& Carvajal (2006) y Rentería (2009).
Para entender los niveles de actuación en las relaciones de trabajo, y en ellas la formación del CP, se toman aquí como marco de referencia y encuadre los componentes de la propuesta de "abordaje psicosocial de la diversidad y papel de formas organizativas en contextos organizacionales instituidos" (p. 149) de Rentería \& Carvajal (2006), como abordaje para el análisis y comprensión de los sistemas organizacionales y las dinámicas de las relaciones de trabajo desde una perspectiva psicosocial.

En esta parte es importante precisar que se asume que las organizaciones de trabajo son entes sociales, artificiales, creados intencionalmente para obtener resultados específicos en relaciones de trabajo y producción (Rentería, 2009). De acuerdo con esta propuesta, la organización, como construcción social, puede ser comprendida por medio del análisis de los niveles desde los cuales se estructura a través de formas organizativas y de los modos como funciona, partiendo de una mirada interaccional-relacional, entendiendo la conformación de un sistema organizacional como un proceso continuo de interacción permanente de actores y agentes sociales que participan de las actividades propias del funcionamiento del sistema (Rentería \& Carvajal, 2006). Así, puede estudiarse no la estructura como resultante estática o prescrita, sino el proceso de estructuración del sistema (Spink, 1996; Weick, 1982), a partir de la identificación y análisis de los niveles de actuación a través de los cuales el sistema se organiza, resulta y se recrea. Los niveles propuestos por Rentería \& Carvajal (2006) para el análisis de un sistema organizacional, se mencionan a continuación.

Inicialmente se encuentra el nivel de lo individual, el cual representa al sujeto como persona y singularidad. Luego, está el nivel de lo ocupacional, que refiere la posición en función de la cual se establece y configura el objeto y objetivo principal de la relación de trabajo que ocupa la persona en su actividad en la organización; este nivel es la razón de ser de las personas en las relaciones de trabajo (Rentería, 2017). El tercer nivel es el de lo divisional, que comprende áreas, procesos funcionales que implican subsistemas de actuación, formas organizativas, a través de los cuales la organización se estructura teniéndolos como referentes o marcos 
cotidianos de actuación. El cuarto nivel es el corporativo que corresponde y orienta a la organización como sistema. Finalmente se encuentra el nivel del entorno, que comprende el medio en el cual la organización actúa, donde tiene influencia, y su existencia es reconocida (Rentería, 2017; Rentería \& Carvajal, 2006).

La lectura desde los niveles de actuación en las organizaciones y relaciones de trabajo se presenta partiendo de la persona que trabaja como singularidad y referente humano primordial, pero debe ser entendida como una opción dinámica y dialéctica que obliga la comprensión de las interfases de las personas con y desde sus entornos, a través de otros referentes y niveles de actuación como los ocupacionales, divisionales y corporativos (Rentería, 2017; Rentería \& Carvajal, 2006).

Estos niveles de actuación y análisis son asumidos aquí como encuadre para la comprensión de las relaciones de trabajo en contextos organizacionales instituidos en los cuales se enmarca la configuración de CP asumiendo la complejidad de las propias relaciones al considerar estos niveles como dimensiones de interacción y relación de orden diverso, reproductores, de mantenimiento o transformadores de repertorios en las propias organizaciones de trabajo (Vesga, 2016).

\section{Lo individual como espacio de la singularidad}

El nivel de lo individual como espacio de la singularidad constituye, además de la síntesis biográfica, la experiencia de la identidad personal, que hace referencia "a ese sentimiento cierto de unicidad, de idiosincrasia y de exclusividad que va acompañado de una sensación de permanencia y continuidad a lo largo del tiempo, del espacio y de las diferentes situaciones sociales [cursivas en el original]" (Pujal, 2004, p. 99), lo que hace al individuo concebirse a sí mismo como un sujeto único, independiente y diferenciado; y al mismo tiempo referente desde el cual se construyen las relaciones con los otros. Este es el nivel donde emerge la conciencia de sí mismo, en tanto individuo que existe como miembro de la especie humana y tiene un lugar en el mundo, con un conjunto de características que particularizan la propia singularidad. De acuerdo con la propuesta de Rentería \& Carvajal (2006), este es el nivel

... del sujeto, del self, de la unidad autoreflexiva que incluye el yo y el mí en el sentido propuesto por Mead (...) [donde] se ubican los llamados procesos psicológicos individuales, los posicionamientos, las decisiones y acciones en tanto que persona humana. Es el nivel de los repertorios de significación y actuación. Es el nivel de la singularidad como persona. (pp. 156-157).

Esta singularidad, además de simbólica y significada, incluye las competencias, conocimientos, actitudes, experiencias, trayectorias, entre otros, desde las cuales las personas forman y reforman sus opciones de dar sentido y actuar en su mundo, incluyendo lo que desarrollan o desarrollarán como actividad transformadora en el hecho social denominado trabajo. Es decir, este es el nivel desde el cual el individuo se posiciona en los contextos de trabajo a partir de sus propios repertorios interpretativos, por medio de los cuales concibe lo que son sus capacidades y potencialidades, así como sus obligaciones hacia los otros y hacia la organización en el marco de las actividades de trabajo que son la base de la constitución del CP.

Este también es el nivel de la formación de esquemas cognitivos por medio de los cuales el individuo interpreta el mundo que le rodea, y es el nivel de la construcción de significados sobre el trabajo, los cuales, según lo refieren Gracia, Martín, Rodríguez \& Peiró (2001), se forman como producto de las experiencias que el sujeto vive en sus contextos de trabajo a partir de las interpretaciones que hace de sus experiencias laborales y trayectorias; en este mismo sentido, Morrison\& Robinson (2004) afirman que en estos mismos marcos de referencia "las personas desarrollan valores generalizados acerca de la justicia, el esfuerzo en el trabajo y la reciprocidad" (p. 165).

En relación con el CP, las formas de significación, las experiencias previas y los procesos de socialización relacionados con el trabajo, constituyen importantes antecedentes que configuran perspectivas des- 
de las cuales el individuo configura sus creencias acerca de los compromisos implícitos en su actual relación laboral como lo plantea Rousseau (2001). En la perspectiva de esta autora, el CP comprende creencias respecto de acuerdos de intercambio entre un individuo y la organización para la cual trabaja; tales creencias, constituyen un aspecto idiosincrásico en la relación de trabajo (Rousseau, 2001). En este nivel la manera como un individuo concibe sus compromisos implícitos en una relación de trabajo está asociado a sus características individuales y al contexto social en que el CP se forma y se mantiene (Linde \& Schalk, 2008). De esta manera, la formación de un CP tiene como uno de sus fundamentos o referentes la singularidad del individuo, su idiosincrasia, su forma de ver e interpretar el mundo.

\section{Lo ocupacional como expresión de roles y posicionamientos}

En este nivel se define la actuación de la persona y su razón de ser como trabajador de la organización e implica la ejecución de las funciones y tareas propias de posiciones ocupacionales que orientan buena parte de las relaciones de trabajo en el sistema organizacional (Rentería, 2017). Este es el nivel en el que se definen roles cuyo origen, según Castells (2004), "se definen por normas estructuradas por las instituciones y organizaciones de la sociedad" (pp. 28-29) e implican la ejecución de uno o más papeles (Goffman, 2009), entendiéndolos como "patrones de comportamiento atribuidos para ciertas posiciones, generalmente dentro de una estructura social” (Bazilli et al., 1998, p. 116), en este caso la organización de trabajo. En este sentido, para un trabajador implica la ejecución de acciones representadas en tareas, funciones y responsabilidades que en general debe cumplir en virtud de la posición que ocupa en un sistema organizacional, lo cual le define su papel y roles ocupacionales.

De acuerdo con Rentería \& Carvajal (2006), este nivel

... corresponde al marco de actuación individual, y en parte a la posición social que estructura, y orienta la actividad de las personas generalmente en función de sistemas clasificatorios como cargos, puestos, responsabilidades o funciones. En este sentido, es el nivel de los papeles [asignados] esperados y asumidos (Bazilli, Rentería, Duarte, Simões, Feitosa, \& Rala, 1998); es el nivel de obtención de marcas y referenciales de identidad [dentro del sistema de trabajo concreto]. (p. 157).

Este es el nivel donde comienzan a configurarse las relaciones de trabajo en cuanto a interacción con los otros, asociada con actividades concretas, prescritas o no, ya que aquí la persona da sentido a su quehacer relacional a partir de la ejecución de una serie de actividades definidas por su rol ocupacional, que le define sus papeles de actuación en la organización, identificables y reconocibles por los "otros" que participan en las dinámicas de trabajo.

Un aspecto clave en este nivel es la configuración de la identidad ocupacional, la cual "se concibe como la relación que las personas tienen con el trabajo respecto a cómo el rol ocupacional (el trabajo que desempeñan) tiene que ver con lo que la persona es, o le describe como parte central suya" (Andrade, 2014, p. 121). De esta manera, el punto de partida de la configuración de las relaciones "de" trabajo es la relación de la persona con "su" trabajo, focalizada en la construcción identitaria de sí mismo como trabajador en un contexto de trabajo, que lo ubica socialmente en el ejercicio de su rol ocupacional. Este rol implica ser y concebirse a sí mismo como miembro de una organización, con un estatus o nivel jerárquico, desde donde concibe o infiere la existencia de un conjunto de compromisos implícitos y explícitos constitutivos de un CP.

Por otro lado, ser miembro de una organización constituye un hecho institucional (Vesga, 2012), el cual está determinado por "la imposición de un status, colectivamente reconocido, al que se vincula una función [o conjunto de funciones]" (Searle, 1997, p. 58). En tal sentido,

...cuando una persona adquiere el estatus de trabajador de una organización, este hecho es colectivamente reconocido por sus miembros; este estatus claramente determina para todos 
los miembros de la organización quién pertenece a ella y quién no, y de este hecho se deriva la condición de que a esta persona se le vinculan ciertas funciones asociadas tanto al cargo [o posición] que ocupa en la organización como a la naturaleza de ella. (Vesga, 2012, p. 83)

En este orden de ideas, ser miembro de una organización en el estatus o condición de trabajador, implica un conjunto de derechos y deberes en relación con la organización, explicitados a través de los acuerdos del contrato jurídico. Sin embargo, más allá de los acuerdos manifiestos las partes "infieren" un conjunto de derechos y obligaciones que no se explicitan en el contrato jurídico pero que se suponen socialmente como parte de la relación de trabajo, los cuales están presentes en la constitución del CP (Vesga, 2012). De esta manera,

... la configuración de un contrato psicológico por parte de una persona en calidad de trabajador de una organización no se da como un hecho aislado de la relación de trabajo sino en el marco de un hecho [social e] institucional, que constituye el establecimiento del carácter de trabajador a partir de los enunciados manifiestos en el contrato de trabajo. Si una persona no pertenece a una organización como trabajador de ésta, podría tener algunas expectativas o deseos de pertenecer a ella, pero tales expectativas y deseos no serían constitutivos de un contrato psicológico. El contrato psicológico, por tanto, sólo se configura en la condición de una relación de trabajo de una persona con una organización (Vesga, 2012, pp. 84-85).

El nivel de lo ocupacional está relacionado también con el tipo o modalidad de vinculación o contratación que tienen los trabajadores con la organización, ya que éstas están asociadas al ejercicio del rol ocupacional y las condiciones de una persona en la misma. De forma complementaria, este nivel está atravesado por las formas de reinstitucionalización del trabajo en la actualidad. Tales modalidades se refieren a contrataciones de carácter indefinido, temporal u ocasional, formas de tercerización como agencias de intermediación, el outsourcing, los servicios profesionales, o formas especiales de contra- tación como el trabajo a domicilio y el teletrabajo (Castel, 2000; Rentería, 2001; 2009; 2012; 2016).

El tipo o modalidad de vinculación laboral está asociado a la configuración de CP como se pone en evidencia en los resultados de algunas investigaciones. Por ejemplo, la contratación de trabajadores temporales impacta negativamente la confianza y el compromiso hacia la organización de parte de los trabajadores permanentes, pues se produce un debilitamiento de la confianza de los trabajadores hacia la organización y una percepción de que su CP ha sido violado (George, 2003). Se ha evidenciado también que el contenido del CP es más amplio en los empleados permanentes que en los temporales (Silla, Gracia, \& Peiró, 2005). Se ha encontrado que existen diferencias importantes en el contenido del $\mathrm{CP}$, dependiendo de la modalidad de trabajo como empleado, outsourcing y Cooperativas de Trabajo Asociado (CTA); en estas formas de trabajo, el CP es más extenso en las modalidades de outsourcing y CTA, y más estrecho en la modalidad de empleo (Vesga, 2007; 2011).

En cuanto a las características del CP en relaciones de empleo contingentes, que implican a los trabajadores contratados, la agencia de empleo y la organización-cliente en cuyas instalaciones trabajan los empleados, se ha evidenciado que el cumplimiento percibido de las obligaciones del cliente se relaciona positivamente con el cumplimiento percibido de las obligaciones de la agencia y que estos conceptos son independientes uno de otro; así mismo, la percepción de inseguridad en el trabajo se relaciona negativamente con el cumplimiento de las obligaciones de la agencia (Chambel \& Fontinha, 2009).

En síntesis, en este nivel se configuran marcos referenciales y operan procesos de construcción de la identidad ocupacional y el ejercicio del rol ocupacional, aspectos que implican la concepción de derechos y deberes por parte del trabajador, en función tanto de la posición que ocupa en el sistema organizacional como del tipo de vinculación contractual que tenga.

Aquí es importante explicitar que, en la perspectiva psicosocial, interactiva y relacional propuesta, las modalidades y condiciones de trabajo como referente de lo ocupacional, configuran simultáneamen- 
te formas organizativas que afectan directamente los repertorios, expectativas, contenidos y extensión del propio CP.

\section{Lo divisional como nivel de estructuración}

Este es el nivel que permite discernir y comprender la manera cómo la organización como sistema se estructura y define a través de divisiones o subsistemas representados en procesos, procedimientos y formas organizativas establecidas en las estructuras oficializadas y declarativas, así como en otras correspondientes a múltiples dimensiones de la vida social, cultural y cotidiana.

De acuerdo con Rentería \& Carvajal (2006), este nivel

... corresponde a las áreas o procesos funcionales que la organización establece para el logro de sus objetivos, así como a los procesos y otras formas de crear subsistemas o partes que participan o existen en la gestión del día a día de las organizaciones. Es decir, es el nivel de la organización oficial y la no oficial, ligado a la manera cómo las personas se organizan para dar vida a la organización (Spink, 1996). Es el nivel de lo estructurante, de las diferentes maneras de dividir o dividirse la organización, de las partes identificables, de los procesos y en parte de la generación de muchas formas organizativas oficializadas o no. (pp. 157-158).

En este nivel, las relaciones de trabajo se conciben en dos dimensiones conocidas como sistema formal e informal (Barnard, 1968; Petit, 1984; Schein, 1994) u oficial y no oficial (Rentería \& Carvajal, 2006).

En la primera dimensión, se encuentran las interacciones dirigidas al desarrollo de las tareas y cumplimiento de los objetivos en el marco de la organización formal (Barnard, 1968) o sistema formal (Petit, 1984), en el cual las relaciones son guiadas por el propósito, objetivos o motivos prescritos más que por aspectos de índole social, cultural o de aspectos de la intimidad de las personas (Bauman \& May, 2007). En esta dimensión, todas las actividades e interacciones implican e inciden en la misión básica de la organización (Schein, 1994).
Esta dimensión incluye la estructura oficial o declarativa de la organización (Rentería \& Carvajal, 2006), en la que las relaciones de trabajo se refieren a las funciones propias de las posiciones ocupacionales, vinculadas en formas organizativas por medio de procedimientos, políticas y reglamentos, expresados en los documentos oficiales de la organización. Aquí existen los denominados "grupos formales" los cuales "son creados deliberadamente por los gerentes con el propósito de desarrollar tareas específicas claramente relacionadas con la misión de la organización" (Schein, 1994, p. 146).

De forma complementaria, la segunda dimensión incluye otras formas de organización en las que las relaciones de trabajo derivadas de interacciones ocurren como producto de afinidades o de conflictos entre los trabajadores y otros actores del sistema organizacional, siendo en el primer caso relaciones consideradas de "compañerismo" que derivan en la constitución de grupos informales 0 no oficiales (Petit, 1984; Rentería \& Carvajal, 2006; Schein, 1994), y en el segundo, "espacios de desencuentro" que originan rupturas y distanciamientos en las relaciones interpersonales, más allá del origen instituido de la relación, o qué tanto aporte al logro del objetivo organizacional. En cualquier caso, éstas influyen y se desprenden de posicionamientos, actitudes y cuestiones disposicionales de las personas involucradas (Barnard, 1968).

En esta dimensión se entiende que las relaciones de trabajo ocurren en un sistema social y cultural (Khanafiah \& Situngkir, 2004), que aunque informal o no oficial, está influenciado también por lo que Hatch (1997) denomina estructura social, entendida como "las relaciones entre los elementos sociales incluyendo las personas, posiciones y unidades organizacionales a las cuales ellos pertenecen" (P. 161). Además de la influencia de la estructura formal, estas relaciones obedecen también a acercamientos y contactos producto de afinidades, coaliciones o agrupamientos transversales entre los sujetos en distintos niveles jerárquicos y áreas de la organización.

Las relaciones de trabajo en el nivel de lo divisional comprenden, en síntesis, el conjunto de interacciones formales e informales, que originan la conformación de colectivos o agrupaciones, de "formas 
organizativas" (Rentería, 2004), ya sean de carácter oficial o no oficial; estas son un aspecto en la dinámica que da vida a las organizaciones a manera de referentes estructurantes. Lo común a todas estas, es que están constituidas por individuos "que se conocen, reaccionan entre sí y están en estado de interdependencia no sólo funcional -por el trabajosino también psicológica [cursivas en el original]" (Petit, 1984, p. 24).

La configuración del CP vista en la perspectiva de este nivel, implica la formación de este en las relaciones diádicas jefe-subalterno, (como se ve por ejemplo en Argyris, 1960) especialmente en el caso de estructuras funcionales en las que el rol ocupacional está definido por la pertenencia del trabajador a una unidad determinada. En el caso de estructuras por procesos o por proyectos, las relaciones de trabajo y los $\mathrm{CP}$ se construyen en función de varios "agentes representantes" de la organización, como ha sido discutido por Rousseau (1995), pues estos "comunican demandas y expectativas relacionadas con el trabajo, promoción y compensaciones" (p. 60), así como sobre las responsabilidades del trabajador en virtud del rol ocupacional que ejerce en un determinado proceso o proyecto.

Así mismo, lo divisional comprende la formación de estructuras relacionales que producen en los trabajadores vinculados a una unidad determinada, procesos de significación de pertenencia a este colectivo dentro del sistema organizacional, en cuyo caso los individuos adscritos a una unidad específica tienden a formar vínculos y experiencias compartidas, percibiéndose como grupo.

En este orden de ideas, Laulié \& Tekleab (2016) han propuesto la existencia de dos niveles de CP en relación con los grupos y equipos de trabajo. De una parte, "las organizaciones también pueden hacer promesas a los equipos como una unidad a cambio de algún tipo de entregables por parte del equipo como unidad" (p. 5), lo que lleva a la configuración de CP entre los equipos y las organizaciones. De otra parte, dado que los miembros de un equipo interactuan y comparten información, la interacción con los miembros de un equipo puede influir en las expectativas que cada individuo forma sobre lo que espera de la organización. En este sentido, se forma lo que los autores denominan un Contrato Psicológico individual compartido, que implica la convergencia de percepciones de los miembros del equipo en relación con los CP individuales (Laulié \& Tekleab, 2016). En tal sentido, los CP tendrán diferencias en sus configuraciones dependiendo no sólo de la singuralidad de cada trabajador como individiduo, sino de su pertenencia a determinada unidad dentro de la organización.

\section{Lo corporativo-integrativo como sistema}

Este nivel constituye la concepción de la organización como una totalidad, como un sistema. De acuerdo con Rentería \& Carvajal (2006),

... es donde se trata de representar la estructura como fijación temporal (Weick, 1982). Es el nivel del intangible que busca mantener la unicidad o totalidad del Contexto Organizacional Instituido, tratando diferenciación y delimitación con su entorno multidimensional pero específico. Es el nivel de las mediaciones simbólicas que dan vida a la organización como "algo" que existe por fuera de las personas que le componen (Spink, 1996, Pagès, 1993) y que facilitan su interiorización por parte de quienes viven y dan vida a ese intangible llamado organización (Enriquèz, E, 2001). Es el nivel de las metáforas (Morgan, 1996) para comprender y explicar la organización como un todo, como un hecho social "tangible". (2006, p. 158).

Las relaciones de trabajo en este nivel se refieren a los nexos de los trabajadores, y de estos, desde sus formas organizativas y grupos de pertenencia o referencia interna o externa, con la organización entendida como una totalidad. Este aspecto implica la construcción de procesos identificación social u organizacional, lo que considera que las personas tienden a clasificarse a sí mismos en categorías sociales, tales como miembros de una organización (Ashforth \& Mael, 1989; Tajfel, 1984).

La identificación social es "la percepción de unidad o pertenencia a algún agregado humano" (Ashforth \& Mael, 1989, p. 21). En cuanto a la identifica- 
ción organizacional, esta representa "la conexión relacional entre los individuos y las organizaciones a las cuales ellos pertenecen" (Parker \& Haridakis, 2008, p. 105). "Es una forma específica de identificación social donde los individuos se definen a sí mismos en términos de su membrecía a una organización en particular" (Mael \& Ashforth, 1992, pág. 105). Como fenómeno psicosocial, es una forma de vínculo que implica la integración de la membrecía organizacional con el autoconcepto personal conectando a los individuos y grupos de referencia con la organización (Parker \& Haridakis, 2008).

Sin embargo, aún en este nivel las relaciones de trabajo son relaciones entre personas y como tales, están sujetas a las mismas restricciones e influencias de otras relaciones humanas; en tal sentido, si la relación se da entre personas, se pregunta Herriot (2001) ¿quién o qué es la organización? "La organización" es un ente abstracto, una generalización que, como totalidad, está constituida por individuos que interactúan y que, en ciertos niveles de ésta establecen pautas de acción, políticas y directrices como "agentes representantes" (Rousseau, 1995) de "la organización" y le dan vida a través de su actividad y sus relaciones. Así, expresiones como "la empresa" o "la organización" generalmente terminan siendo una forma de "generalización" de las pautas de acción emanadas de las personas que ejercen roles directivos y desde donde se definen las políticas, normas y directrices que rigen el desempeño de los trabajadores.

De esta manera, se entiende que los individuos no sólo establecen relaciones con otros sujetos como individuos o con grupos o formas organizativas de las que forma parte en sus contextos laborales, sino que lo hacen también con la organización misma como una unidad totalizada. Así, para cada trabajador la organización tiene y representa un elemento referencial, un predicado (Malvezzi, 2000) de una identidad definida, la "que permite distinguir a cada organización como singular, particular y distinta de las demás" (Etkin \& Schvarstein, 2000, p. 51).

La concepción que tiene un trabajador de la organización como una totalidad, se construye a través de procesos de socialización. Cuando un individuo se vincula a una organización en calidad de trabajador, independientemente de la modalidad de contrato jurídico que tenga, ya sea como empleado directo de la empresa o en modalidades de tercerización, requiere de un proceso mínimo de socialización particularizada -generalmente denominada Inducciónque tiene el propósito de dar a conocer e incorporar las normas que en general regulan el funcionamiento de ese sistema de la cual ahora forma parte, para poder interactuar de manera efectiva en el desempeño de su rol como trabajador.

Este proceso de socialización -o "culturización” si se quiere- comprende la internalización de un conjunto de pautas de comportamiento que, al asumirlas, le permiten al individuo ser miembro de una sociedad que preexiste a él y a la cual él ahora se incorpora para formar parte de ella. En la perspectiva de Berger \& Luckmann (1995) se trata de un proceso de socialización secundaria como "proceso que induce al individuo ya socializado a nuevos sectores del mundo objetivo de su sociedad" (p. 166) como es el caso del ingreso de un individuo a una organización como miembro de esta. Es decir, en una perspectiva interaccionista amplia, se trata de la incorporación de patrones o pautas de un "recién llegado" a un contexto instituido de relaciones de trabajo, en este caso llamado organización o empresa.

La formación del CP vista desde este el nivel de lo corporativo, ha sido la perspectiva que ha estado presente en la mayor parte de la teorización e investigación sobre este concepto, entendiéndolo como el conjunto de expectativas y creencias sobre obligaciones mutuas entre un trabajador y la organización.

En este nivel, las partes intervinientes en la conformación de un CP son el trabajador y agentes representantes de la organización en niveles directivos, como lo plantean Rousseau (1995) y Guest (2004), puesto que la organización, siendo un ente abstracto, no puede concebir compromisos implícitos, es decir, un CP como lo afirman Conway \& Briner (2005).

En este orden de ideas, es clave el papel que juegan los procesos o áreas de Recursos Humanos, 
ya que las políticas y directrices que se establecen desde allí impactan directamente la gestión y desempeño de los trabajadores y constituyen un elemento fundamental para la comprensión de la relación de los trabajadores con la organización (Aggarwal \& Bhargava, 2009), específicamente aquellas como reclutamiento, selección e inducción, entrenamiento y desarrollo, evaluación del desempeño y compensaciones (Wangithi \& Muceke, 2012).

\section{El entorno como marco de actuación organizacional}

El nivel del entorno se refiere al "campo de actuación y reconocimiento de la existencia social de la organización como tal” (Rentería \& Carvajal, 2006, p. 158). Es el contexto constituido por aspectos generales y específicos de orden social, cultural, político, económico, jurídico y ambiental, en el cual la organización existe y actúa como un conjunto o sistema abierto y en el que produce una singularidad que le permite ser reconocida e identificada como tal.

Este es también el lugar de los contratos sociales que pautan los acuerdos de orden macro en los cuales se insertan los acuerdos y relaciones que inciden de manera transversal (Rentería, 2017; Rentería \& Carvajal, 2006) en los demás niveles de actuación. La idea de pactos o acuerdos de orden social que establecen las constituciones de las sociedades organizadas y el gobierno de los pueblos fue propuesta por Rousseau, J. J. (2007) quien en su obra el contrato social ${ }^{1}$ da descripción definida de lo que constituye este hecho en la conformación de las sociedades.

Esta concepción de "pacto social" implica que los hombres constituyan una forma de asociación, una suma de fuerzas para conservarse y subsistir como especie humana (Rousseau, 2007). Esta visión de un contrato social se inscribe en la perspectiva de acuerdos sociales colectivos orientados al bien común, los cuales se fundamentan principalmente en aspectos éticos y se constituyen a partir normas socialmente compartidas y aceptadas.
Respecto de las relaciones de trabajo, este nivel constituye marcos de referencia social y culturalmente amplios que delinean pautas de comportamiento sobre las actuaciones válidas en las interacciones sociales, adquiridas a través de procesos de socialización primaria y secundaria (Berger \& Luckmann, 1995) y que son legitimadas por y en el contexto e interfase Entorno-Organización. Es el lugar de las normas sociales que regulan la conducta aceptada o esperada de acuerdo con los roles que se desempeñan y posiciones que se ocupan (Myers, 1991; Rentería \& Carvajal, 2006). Es el nivel de las instituciones, entendidas como "aquellos cuerpos normativos jurídico-culturales compuestos de ideas, valores, creencias, leyes que determinan las formas de intercambio social" (Schvarstein, 1991, p. 26), y de lo instituido, lo cual constituye "aquello que está establecido, el conjunto de normas y valores dominantes, así como el sistema de roles que constituye el sostén de todo orden social" (Schvarstein, 1991, p. 26).

En relación con la configuración del $\mathrm{CP}$ en la perspectiva de este nivel, puede decirse que las inferencias que, las partes que participan de la relación de trabajo, hacen sobre los derechos, obligaciones, expectativas e intercambios producto de su relación laboral, se derivan a su vez de los significados construidos en el sistema social más amplio en el que la organización está inserta y que se refieren a los supuestos sobre derechos y obligaciones de las partes implicadas en una relación.

Tales compromisos, expectativas e intercambios implícitos que constituyen el $\mathrm{CP}$, son emanados del contexto en la que las partes de la relación de trabajo están inmersas, debido a que las relaciones de trabajo se configuran en niveles sociales más amplios. En este sentido, los CP son también acuerdos micro-sociales que se corresponden con pactos sociales de orden macro. Como lo sugiere Barnard (1968), en muchos casos las relaciones contractuales específicas involucran no sólo circunstancias legales formales sino actitudes y prácticas habituales de carácter social más general.

$1 \quad$ Publicado originalmente en 1762 


\section{A modo de conclusión}

Las organizaciones son sistemas sociales complejos en los que la relación permanente entre sus miembros genera dinámicas y patrones de interacción y comunicación en diversos niveles jerárquicos, diadas, grupos y subsistemas, con miras a la realización de las tareas propias de los cargos y áreas de gestión, en virtud de las posiciones y roles que ocupan las persona en el desarrollo de su trabajo.

Paralelamente, ya sea de forma sincrónica o asincrónica, los miembros de una organización establecen también, además de las relaciones formales asociadas a los objetivos laborales, interacciones sociales de carácter informal, en lo que se construyen vínculos de afinidad o disparidad entre las personas (Barnard, 1968). En estas dinámicas, los miembros de una organización construyen significados y sentidos sobre su trabajo, su identidad laboral, la organización, y, fundamentalmente, sobre las relaciones de trabajo, que constituyen un aspecto central de su cotidiano laboral.

Las relaciones de trabajo son complejas y se configuran en diversos niveles de interacción, lo que se ha denominado como los niveles de actuación en las relaciones de trabajo (Rentería \& Carvajal, 2006).

En el marco de las relaciones y niveles de actuación, se construyen los $\mathrm{CP}$, y dada la complejidad de las interacciones sociales en contextos de trabajo, se sigue que es necesario adoptar una mirada multidimensional del CP (Vesga, 2017), en el sentido de que existen aspectos clave en lo individual, lo ocupacional, lo divisional, lo corporativo y lo contextual de las dinámicas de trabajo, que aportan elementos para la configuración de los CP y comprensión de estos.

El CP implica compromisos implícitos en las relaciones de trabajo, y desde la mirada aquí propuesta, tales compromisos están afectados tanto por las trayectorias personales de los individuos en el trabajo como por el ejercicio de sus roles laborales. Así mismo, la inferencia de compromisos implícitos, no se da sólo de manera diádica entre trabajadores y jefes u organizaciones, tal como ha sido presentado hasta ahora en la literatura sobre el $C P$, sino que se suponen también compromisos en relación con la pertenencia a divisiones de la organización o grupos de trabajo.

\section{Referencias}

Aggarwal, U. \& Bhargava, S. (2009). Reviewing the relationship between human resource practices and psychological contract and their impact on employee attitude and behaviours: A conceptual model. Journal of European Industrial Training, 33(1), 4-31. Doi: https:// doi.org/10.1108/03090590910924351

Alcover, C. M. (2002). El contrato psicológico: el componente implícito de las relaciones laborales. Málaga: Aljibe.

Alcover, C.-M., Rico, R., Turnley, W., \& Bolino, M. (2016). Understanding the changing nature of psychological contracts in 21st century organizations: Amultiple-foci exchange relationships approach and proposed framework. Organizational Psychology Review, 7(1) 4-35. Doi: https://doi.org/10.1177/2041386616628333

Andrade, V. (2014). Identidad profesional y el mundo del trabajo contemporáneo. Reflexiones desde un resumen de caso. Athenea Digital, 14(2), 117-145.

Argyris, C. (1960). Understanding Organizational Behavior. Homewood: Dorsey.

Ashforth, B. E. \& Mael, F. (1989). Social Identity Theory and the Organization. Academy of Management Review, 14(1), 20-39. Doi: https:// doi.org/10.5465/amr.1989.4278999

Barnard, C. (1968). The Functions of the Executive. Cambridge: Harvard.

Bauman, Z. \& May, T. (2007). Pensando sociológicamente ( $2 \mathrm{a}$ ed.). Buenos Aires: Nueva Visión.

Bazilli, C., Rentería, E., Duarte, J. C., Simões, K. V., Feitosa, L., \& Rala, L. A. (1998). Interacionismo simbólico e teoría dos papéis. Uma aproximação para a psicologia social. São Paulo: EDUC.

Berger, P. \& Luckmann, T. (1995). La construcción social de la realidad. Buenos Aires: Amorrortu.

Castel, R. (2000). As transformações da questão social. En R. Castel, L. E. Wanderley, \& M. 
Belfiore-Wanderley, Desigualdade e a questão social (pp. 235-264). São Paulo: Educ.

Castells, M. (2004). La Era de la Información: Economía, Sociedad y Cultura. Vol. 2. El poder de la identidad (5a ed.). México, D.F.: Siglo XXI.

Chambel, M. J. \& Fontinha, R. (2009). Contingencies of Contingent Employment: Psychological Contract, Job Insecurity and Employability of Contracted Workers. Revista de Psicología del Trabajo y de las Organizaciones, 25(3), 207-217. Doi: https://doi.org/10.4321/ s1576-59622009000300002

Conway, N. \& Briner, R. (2005). Understanding Psychological Contracts at Work: A critical evaluation of theory and research. New York: Oxford. Doi: https://doi.org/10.1093/acprof:o so/9780199280643.001.0001

Coyle-Shapiro, J. \& Parzefall, M. (2010). Psychological contracts. Fuente: LSE Research Online: http://eprints.lse.ac.uk/26866/

Etkin, J. \& Schvarstein, L. (2000). Identidad de las organizaciones. Invariancia y cambio. Buenos Aires: Paidós.

George, E. (2003). External Solutions and Internal Problems: The Effects of Employment Externalization on Internal Workers' Attitudes. Organization Science, 14(4), 386-402. Doi: https://doi.org/10.1287/orsc.14.4.386.17488

Goffman, E. (2009). La presentación de la persona en la vida cotidiana ( $2 a$ ed.). Buenos Aires: Amorrortu.

Gracia, F. J., Martín, P., Rodríguez, I., \& Peiró, J. M. (2001). Cambios en los componentes del significado del trabajo durante los primeros años de empleo: Un análisis longitudinal. Anales de Psicología, 17(2), 201-217.

Guest, D. (2004). The Psychology of the Employment Relationship: An analysis based on the Psychological Contract. Applied Psychology, 53(4), 541-555. Doi: https://doi. org/10.1111/j.1464-0597.2004.00187.x
Hatch, M. J. (1997). Organization Theory. New York: Oxford. Doi: https://doi.org/10.1287/ orsc.8.3.275

Herriot, P. (2001). The Employment Relationship: A Psychological Perspective. Philadelphia: Routledge.

Khanafiah, D. \& Situngkir, H. (2004). Social Balance Theory. Fuente: http://cogprints.org/3641/

Laulié, L. \& Tekleab, A. G. (2016). A MultiLevel Theory of Psychological Contract Fulfillment in Teams. Group \& Organization Management, 41(5), 1-41. Doi: https://doi. org/10.1177/1059601116668972

Linde, B. \& Schalk, R. (2008). Influence of pre-merger employment relations and individual characteristics on the psychological contract. South African Journal of Psychology, 38(2), 305-320. Doi: https://doi.org/10.1177/008124630803800204

Mael, F. \& Ashforth, B. (1992). Alumni and their alma mater: A partial test of the reformulated model of organizational identification. Journal of Organizational Behavior, 13(2), 103-123. Doi: https://doi.org/10.1002/job.4030130202

Malvezzi, S. (2000). A construção da identidade profissional no modelo emergente de carreira. Organizações \& Sociedade, 7(17), 137-143. Doi: https://doi.org/10.1590/ S1984-92302000000100011

Malvezzi, S. (2011). A Gestão dos Contratos Psicológicos. Revista do Instituto de Marketing Industrial, 53, 66-73.

Morrison, E. \& Robinson, S. (2004). The Employment Relationship from Two Sides: Incongruence in Employees' and Employers' Perceptions y Obligations. En J. A.-M. Coyle-Shapiro, L. M. Shore, M. S. Taylor, \& L. E. Tetrick (Eds.), The Employment Relationship. Examining Psychological and Contextual Perspectives (pp. 161-180). New York: Oxford.

Myers, D. (1991). Psicología Social (2a ed.). Madrid: Panamericana. 
Parker, R. \& Haridakis, P. (2008). Development of an Organizational Identification Scale: Integrating congnitive and communicative conceptualizations. Journal of Communication Studies, 1(3/4), 105-126.

Petit, F. (1984). Psicosociología de las Organizaciones. Barcelona: Herder.

Pujal, M. (2004). La identidad (el self). En T. Ibañez, M. Botella, M. Domènech, J. Feliu, L. M. Martínez, C. Pallí, M. Pujal, F. J. Tirado, Introducción a la psicología social (pp. 93-138). Barcelona: UOC.

Rentería, E. (2001). El modelo educativo tradicional y los perfiles de competencias según las modalidades y tendencias de trabajo actuales. En Asociación Inroamericana de Postgrado, Vinculación universidad-estado a través del postgrado: pautas y lineamientos ( $\mathrm{pp}$. 5161). Barcelona: Asociación Iberoamericana de postgrado.

Rentería, E. (2004). De las intervenciones grupales a las intervenciones Sociales. Un ensayo sobre el uso del conocimiento científico en el caso de las intervenciones profesionales. Revista de Estudios Sociales, 18, 37-49.

Rentería, E. (2008). Nuevas realidades Organizacionales y del Mundo del Trabajo: Implicaciones para la Construcción de la Identidad o del Sujeto. Informes Psicológicos, 10(10), 65-80.

Rentería, E. (2009). De recursos humanos a la Psicología Organizacional y del Trabajo. Reflexiones a la luz de las realidades actuales del mundo del trabajo. En M. C. Aguilar, \& E. Rentería (Eds.), Psicología del trabajo y las organizaciones: reflexiones y experiencias de investigación (pp. 25-52). Bogotá D. C.: Universidad Santo Tomás.

Rentería, E. (2012). Desarrollo de la empleabilidad como estrategia para las organizaciones: limitaciones, posibilidades e implicaciones para las personas. Aristeo, 2, 69-90.

Rentería, E. (2016). Salud, bienestar y mundo del trabajo. Entre lo normativo, los riesgos, y la coexistencia de realidades. En M. L. Costa, \& J. A. Zanatta, Psicologia da Saúde: discussões temáticas (pp. 171-192). Campo Grande: UCDB.

Rentería, E. (2017). Psicologías del trabajo y Organizacionales. Una re-introducción desde la reinstitucionalización actual de las relaciones de trabajo. Cali: Universidad del Valle.

Rentería, E. \& Carvajal, B. (2006). Abordaje psicosocial de la diversidad y papel de formas organizativas en contextos organizacionales instituidos. Psicología desde el Caribe, 17, 149-175.

Rousseau, D. (1995). Psychological Contracts in Organizations: Understanding Written and Unwritten Agreements. Thousand Oaks: Sage.

Rousseau, D. (2001). Schema, promise and mutuality: The building blocks of the psychological contract. Journal of Occupational and Organizational Psychology, 74, 511-541. Doi: https://doi.org/10.1348/096317901167505

Rousseau, J. J. (2007). Contrato Social (12a ed.). Madrid: Espasa Calpe.

Schein, E. (1994). Organizational Psychology (3rd ed.). New Jersey: Prentice-Hall.

Schvarstein, L. (1991). Psicología social de las organizaciones. Nuevos aportes. Buenos Aires: Paidós.

Searle, J. R. (1997). La construcción de la realidad social. Barcelona: Paidós.

Silla, I., Gracia, F. J., \& Peiró, J. M. (2005). Diferencias en el contenido del contrato psicológico en función del tipo de contrato y de la gestión empresarial pública o privada. Revista de Psicología Social, 20(1), 61-72. Doi: https:// doi.org/10.1174/0213474052871097

Spink, P. (1996). A organização como fenômeno psicossocial: notas para uma redefinição da psicologia do trabalho. Psicologia \& Sociedade, 8(1), 174-192.

Spooner, K. \& Haidar, A. (2006). Defining the Employment Relationship. International Journal of Employment Studies, 14(2), 63-82. 
Tajfel, H. (1984). Grupos humanos y categorías sociales. Barcelona: Herder.

Topa, G. (2005). El contrato psicológico: un nuevo marco para comprender las relaciones dentro de las organizaciones. En F. J. Palací, Psicología de la organización (pp. 101-119). Madrid: Pearson.

Vesga, J. J. (2007). Contenido del contrato psicológico percibido en distintas modalidades de trabajo. Perspectivas en Psicología(10), 79-99.

Vesga, J. J. (2009). El contrato psicológio y las nuevas realidades del trabajo. En M. C. Aguilar, \& E. Rentería (Eds.), Psicología del trabajo y de las organizaciones: reflexiones y experiencias de investigación (pp. 99-110). Bogotá, D.C.: Universidad Santo Tomás.

Vesga, J. J. (2011). Los tipos de contratación laboral y sus implicaciones en el contrato piscológico. Pensamiento Psicológico, 9(16), 171-182.

Vesga, J. J. (2012). El contrato psicológico y la pragmática. En S. Malvezzi, J. J. Orejuela, R. M.
Chiuzi, J. J. Vesga, \& W. A. Riascos, Gramáticas actuales de la relación hombre-trabajo: propuestas de lectura (pp. 75-87). Cali: Editorial Bonaventuriana.

Vesga, J. J. (2016). El contrato psicológico: una propuesta de lectura en el marco de las relaciones de trabajo (Tesis doctoral). Cali: Universidad del Valle.

Vesga, J. J. (2017). El contrato psicológico: un concepto multidimensional. En E. Rentería (Comp.), Entre lo disciplinar y lo profesional. Panorama de experiencias en psicología organizacional y del trabajo en iberoamérica (pp. 297-311). Cali: Universidad del Valle.

Wangithi, W. E. \& Muceke, N. J. (2012). Effect of Human Resource Management Practices on Psychological Contract in Organizations. International Journal of Business and Social Science, 3(19), 117-122.

Weick, K. E. (1982). Psicología social del proceso de organización. Bogotá D. C.: Fondo Educativo Interamericano. 